\title{
Herbal and Alternative Medicine News
}

Green tea molecule could prevent heart attacks

Date: May 31, 2018

Source: British Heart Foundation

Scientists have discovered that a compound found in green tea, currently being studied for its ability to reduce amyloid plaques in the brain in Alzheimer's disease, also breaks up and dissolves potentially dangerous protein plaques found in the blood vessels.

Nanoparticles derived from tea leaves destroy lung cancer cells: Quantum dots have great potential

Date: May 21, 2018

Source: Swansea University

Nanoparticles derived from tea leaves inhibit the growth of lung cancer cells, destroying up to 80 percent of them, new research has shown. The team made the discovery while they were testing out a new method of producing a type of nanoparticle called quantum dots. These are tiny particles which measure less than 10 nanometers. A human hair is 40,000 nanometers thick.

Reishi mushroom powder is an effective treatment for mouth ulcers

Published Date: 07/17/2018, By Edsel Cook

The medicinal mushroom known as "Reishi " in Japan was the subject of a recent Chinese study on alternative treatments for recurrent oral ulceration (ROU). The results showed that freeze-dried reishi mushroom powder provided effective treatment for the most prevalent mouth ulcer disease in the world. ROU is considered an autoimmune disease. It is a frequent complication for acquired immune deficiency syndrome (AIDS) and other diseases that undermine the immune system.

Asafoetida, a plant used in traditional folk medicines, found to show antitumor effects on breast cancer

Published Date: 07/17/2018, By Ralph Flores

Information Collected and complied by:

Md. Akbar Hossain

Department of Pharmacy

ASA University Bangladesh
Plants and herbs that have long been used in folk medicine may very well hold the key to treating modern-day diseases. In a study published in the Journal of Ayurveda and Integrative Medicine, researchers found that asafoetida, a plant used in traditional food and medicine, could potentially have anti-tumor and anti-metastasis effect against breast cancer. The study, led by researchers from Shahid Sadoughi University of Medical Sciences and Ahvaz Jundishapur University of Medical Sciences in Iran, looked at the effect of an oleo gum resin from asafoetida in mice induced with breast cancer.

Herbal plants found to treat tuberculosis naturally

Published Date: 07/17/2018, By Rhonda Johansson

A major issue facing the medical community today is the rise of multidrug-resistant tuberculosis, which hampers current efforts to end the deadly disease globally. The World Health Organization (WHO) estimates that while the incidences of tuberculosis have been decreasing by around two percent each year, all effort must be made in exploring every option to treat it.

This includes validating well-known traditional ethnomedicines. It was with this goal that researchers analyzed the extracts of crepe jasmine (Tabernaemontana coronaria), lemongrass (Cymbopogon citratus) and crepe ginger (Costus speciosus). These plant materials have been traditionally used as remedies for various symptoms of tuberculosis.

Researchers evaluated the three plant extracts for their anti-tuberculosis activity. Part of their analysis included assessing which phytochemical compounds were the most active in influencing the growth kinetics and cellular integrity of a tubercle organism.

Source: alternativemedicine.news 